\title{
Les premiers imprimés religieux au Canada 1764 - 1800
}

\section{Marcelle G. Gingras}

Volume 35, 1968

URI : https://id.erudit.org/iderudit/1007308ar

DOI : https://doi.org/10.7202/1007308ar

Aller au sommaire du numéro

Éditeur(s)

Les Éditions Historia Ecclesiæ Catholicæ Canadensis Inc.

ISSN

0318-6172 (imprimé)

1927-7067 (numérique)

Découvrir la revue

Citer cet article

Gingras, M. G. (1968). Les premiers imprimés religieux au Canada 1764 - 1800 Sessions d'étude - Société canadienne d'histoire de l'Église catholique, 35, 73-79. https://doi.org/10.7202/1007308ar

Tous droits réservés @ Les Éditions Historia Ecclesiæ Catholicæ Canadensis Inc., 1968
Ce document est protégé par la loi sur le droit d'auteur. L'utilisation des services d’Érudit (y compris la reproduction) est assujettie à sa politique d'utilisation que vous pouvez consulter en ligne.

https://apropos.erudit.org/fr/usagers/politique-dutilisation/ 


\section{Les premiers imprimés religieux au Canada 1764 - 1800}

Sous le régime français, il n'y avait pas d'imprimerie au Canada, ni de commerce du livre. Cependant, la Nouvelle-France n'eut pas à souffrir de cette situation. Les inventaires de notaires et les ventes de successions prouvent qu'il y avait au pays des volumes en quantité suffisante pour les besoins de l'époque.

Il est vrai que les bibliothèques les mieux habitées étaient celles des religieux, ce qui explique la quantité et l'utilité des lectures. Mais à mesure que le développement de la colonie prospèrera et que les échanges avec la France deviendront plus faciles, les navires français transporteront les livres commandés non seulement par la religion, mais aussi par l'amitié et l'intérêt. Sans oublier ceux que les voyageurs prévoyants mettront dans leurs bagages. De sorte qu'il y aura une plus grande variété de lecture.

Benjamin Sulte fixe à 60,000 le nombre approximatif de livres qu'on pouvait trouver au Canada, pour une population de 50,000 habitants, en 1765 .

Sans mettre en doute la véracité d'un registre du temps, qui mentionne un nommé Nicolas surnommé "Le liseux", on peut conclure que les livres étaient encore plus abondants que les heures de loisir dont les premiers Canadiens disposaient pour lire.

Mais avec la Conquête, et les guerres qui la précédèrent, le Canada dut ajouter aux deuils et à la destruction qui accompagnent toujours les batailles, la rareté des livres, surtout religieux, et connaître d'insurmontables difficultés pour s'en procurer sous les lois d'un vainqueur, de langue et de religion différentes de celles du pays conquis.

Le temps était venu de reformer l'enseignement religieux et profane désorganisé par les hostilités.

Des centaines d'enfants étaient parvenus à l'âge de "marcher au catéchisme» jusqu'à la première communion et pour les préparer à recevoir cet important sacrement, il fallait l'étude du catéchisme, manuel qui n'existait plus. Les livres d'instruction religieuse qui avaient échappé aux incendies étaient usés et ne pouvaient être remplacés.

1 C. F. R. Fauteux, p. 13 
Monseigneur de Saint-Vallier qui recommandait aux familles chrétiennes " même à celles qui ne savaient pas lire, de garder en leurs maisons plusieurs exemplaires de catéchisme " n'aurait pas pu, en 1764, en trouver même pour les familles qui savaient lire.

On éprouvait d'autant plus le besoin de se procurer des livres nécessaires à l'éducation des enfants que l'autorité religieuse était convaincue que de "l'Instruction des jeunes dépendait la conservation de la $\mathrm{Foi}_{\mathrm{i}}$ et des bonnes mœurs ».

Instruire à cette époque, consistait à apprendre à prier en apprenant à lire, à compter et à chanter le grégorien, et se préparer au service des autels. Mais même réduit à cette proportion, l'enseignement a besoin de manuels.

Depuis la conquête, le Canada devenu possession anglaise, se voyait interdire tout commerce direct avec la France. C'est, écrira M. Villars, " les hostilités de mer entre la France et l'Angleterre ${ }^{2}$ ». On pensa alors à s'approvisionner par Londres, en confiant à des agents britanniques les commandes pour Paris. Ces agents les ayant reçues au port londonien, les auraient ensuite acheminées vers le Canada.

Mais il sera impossible de profiter de ce long détour via Londres. A preuve, du Séminaire des Missions Étrangères de Paris, on écrivait, le 10 avril 1764, au Séminaire de Québec: "Je ne sais, monsieur, s'il sera prudent ni même guères possible de vous faire passer les livres classiques que vous $m$ 'avez demandés. M. Montgolfier m'a dit, ainsi, que les catéchismes sont marchandises de contrebande, en Angleterre ${ }^{3}$. :

L'interdiction sur l'importation et le commerce du livre religieux catholique ne laisse pas d'issue, pour le moment, au renouvellement des manuels scolaires.

C'est à ce moment propice que William Brown vient introduire l'imprimerie au Canada. Né en Écosse, il avait appris son métier en Virginie, et s'était associé à Thomas Gilmore qu'il avait chargé d'acheter, en Angleterre, le papier, la machinerie et les caractères nécessaires pour l'installation de Québec. Gilmore qui n'avait pas seulement le don d'arriver au bon moment, était aussi prévoyant, puisqu'il avait eu la bonne pensée de s'abonner à plusieurs périodiques anglais, avant son départ pour le Canada, dans le but de se procurer des nouvelles pour la Gazette qu'il se proposait d'éditer à Québec. Non moins clairvoyant, Brown avait apporté de Philadelphie un prospectus destiné à solliciter des abonnements et des annonces, pour le journal à paraître. Ayant établi leur atelier à la Haute-Ville de Québec, sur la rue Saint-Louis, ils

2 Lettres M. 163 M. Villars A.S.Q.

3 Lettre M.122 M. VILlars A.S.Q. 
se mirent à l'œuvre rapidement et lancèrent leur premier numéro de la Gazette, le 21 juin 1764, en anglais et en français. C'est à ces deux imprimeurs anglais et protestants que vont se confier les catholiques pour renouveler leurs livres scolaires et leur littérature religieuse. Comme à de rares exceptions près, on n'a toujours que les convictions de ses intérêts, les deux premiers imprimeurs protestants acceptèrent la bonne fortune que leur apportaient ces messagers du ciel.

Le premier livre français et catholique imprimé au Canada, portait le titre de Catéchisme de Sens, nom du diocèse dont Mgr Languet, auteur du texte, avait été l'archevêque. C'était une copie du livre publié à Soissons, en 1727 et entré au pays en 1751. Ce manuel de 177 pages avait été réimprimé à Québec, le 21 novembre 1765, pour la première fois sans imprimatur, parce que Québec était sans évêque et par la volonté de l'Angleterre. Il remplaçait le catéchisme de Mgr de Saint-Vallier. Le premier tirage se fit à 2,000 exemplaires et coûtait $\$ 306$. En moins de deux ans, en avril 1766 , on en commandait une seconde édition de 148 pages, différente et au même prix.

L'édition de 1777 fut approuvée par Jean Olivier Briand, nouvel évêque de Québec, surintendant de l'Église Catholique pour l'Angleterre et ses représentants. L'évêque reconnaissait comme officiel le Catéchisme de Sens remanié, qui portait cette fois l'imprimatur et un mandement qui sanctionnait cette reconnaissance.

De 1764 à 1800 , il n'y eut pas moins que 10 impressions de catéchismes, petits et grands, comme on les désignait. Les uns furent imprimés à Québec, les autres à Montréal qui avait son imprimerie depuis 1776, soit 12 ans après Québec. Le propriétaire en était Fleury Mesplet, né à Lyon, qui a laissé la réputation d'un athée.

Dans la langue anglaise, il y eut le catéchisme dit « de Douay ", en 1778. Imprimé à 1000 exemplaires, le texte avait été préparé par Henry Tuberville, en 1649. Il fut réédité en 1800 , à la demande d'une centaine de familles irlandaises catholiques, sous le nom de Le compagnon $d u$ Catholique sincère, et était distribué gratuitement aux familles pauvres ${ }^{4}$.

Cependant, le livre religieux le plus remarquable que Brown et Gilmore aient sorti de leur presse, est sans doute le catéchisme composé en montagnais et en dialecte algonquin, et préparé par le missionnaire jésuite Jean-Baptiste de la Brosse, en 1767. Sous le titre Nehiro Irénui Nasinahigan, il fut édité à 2000 exemplaires. Plus tard, un alphabet et un calendrier composés dans la même langue atteignaient 3000 copies. C. Imprints.

4 The Sincere Catholiks Compagnion, Marie Tremaine - Bibliography of 
Le record de production des manuels de catéchisme fut atteint le 5 avril 1792, par l'imprimeur Samuel Neilson. 6000 exemplaires de catéchisme double étaient vendus, tandis que les petits catéchismes ne se vendaient qu'à 3000 , le 26 février 1791 .

Le catéchisme ne pouvait suffire à combler les besoins religieux et conme l'interdiction anglaise s'appliquait à toute littérature catholique, on demandera à l'imprimerie locale, de fournir la substance nécessaire aux appétits spirituels.

En 1769, paraît Heures de Vie, tiré à 800 exemplaires. Chez le même libraire, Joseph Bargeas, installé rue Couillard, à Québec, on offre une Neuvaine à saint François-Xavier - à ce saint patron du premier évêque du Canada, Mgr de Laval.

Le livre contenait 215 pages et était imprimé par Brown et Gilmore. L'édition sera renouvelée à Montréal, en 1778, par Mesplet qui en fera une seconde édition, en 1796. Ce qui dénote la grande fareur dont jouissait ce saint, en terre canadienne.

Un autre ouvrage de piéte : La dévotion aux saints Anges Gardiens, se limitera à 84 pages.

Un Exercice très Dévot envers Saint-Antoine de Padoue, préparé par le frère Alexis Dumonceau, sera aussi ajouté au menu des âmes pieuses. En 1774, Brown, dont l'associé Gilmore est décédé, publie une première série de Psautier de David. On l'imprimera aussi à Montréal, et de nouveau, à Québec, en 1785. C'était le manuel scolaire à caractère religieux qui constituait, avec l'abécédaire et le a livre pour apprendre à bien lire ", le contenu du cartable d'un jeune écolier. Les Actes des Apôtres, œuvre de Samuel Neilson, paraît en anglais et en français, en 1791, accompagné de La Journée du Chrétien sanctifié par la prière et la méditation.

La vie abrégée de la Très Sainte Vierge, paraît le 15 septembre 1797, suivie de : Le petit livre de vie, par le jésuite Amable Bonafous.

La Confrérie de l'Adoration Perpétuelle du Saint-Sacrement et de la bonne mort, érigée en l'église paroissiale de Ville-Marie. offre son opuscule qui contient une méditation pour tous les jours de l'année, et de la matière à satisfaire les plus exigeants dévots du temps. Ajoutons encore le Formulaire de Prières, en usage chez les Ursulines et chez leurs élèves. Mesplet en donne une édition en 1777, et la Nourelle Imprimerie de Québec en donne une autre, en 1799. C'était un livre de 500 pages.

La dévotion à la Sainte-Famille établie au Canada, en 1650, par Mgr de Laval est toujours vivace, en 1787. Mesplet édite : Solide dévotion à la Sainte Famille de Jésus, Marie, Joseph. 
De Pierre Hubert, paraît Instructions chrétiennes pour jeunes gens utiles à toutes sortes de personnes.

Toujours de la même source d'inspiration, est publié par Mesplet, en 1777, de même qu'à la Nouvelle Imprimerie de Québec, le Petit Livre de Vie. Ce volume portait une illustration du Christ, innovation de l'imprimerie québecoise. La première édition de ce Petit Livre comptait 456 pages auxquelles il en sera ajouté 100, à la seconde commande. Ce qui fera un Petit Livre de 556 pages... Pour une foi qui se dit capable de transporter les montagnes, un livre de 556 pages peut bien se qualifier " petit ".

Pour terminer cette catégorie, mentionnons: La prière de la congrégation. C'était une supplique spéciale ayant pour sous-titre: - Élection de Saint-Joseph pour préfet perpétuel de la Congrégation de Ville-Marie ». Le besoin exigea qu'on fournisse aussi des livres liturgiques. $400 \mathrm{Graduel}$ Romain furent édités par John Neilson.

En 1795, suit Le livre des heures Romaines, où se trouvaient les psaumes, hymnes et antiennes de l'Église.

En 1777, à la demande de Mgr Briand, les Sulpiciens avaient préparé l'impression d'une messe en l'honneur du sacerdoce de N.S. Jésus-Christ, qui fut publiée à 600 copies distribuées au clergé, par l'évêque de Québec.

L'Office de la Semaine Sainte, en 420 pages, missel pour les fidèles, parut en 1778, chez Mesplet, qui avoue l'avoir copié sur la meilleure édition de Paris.

Parmi les ouvrages suggérés aux paroissiens pour réveiller leur ferveur, il y eut les manuels de cantiques, dont le premier fut : Cantiques de l'Âme Dévote, chez Mesplet, en 1776. C'était une réédition des Cantiques de Marseille, publiés en France, à partir de 1678, sur des airs populaires.

Mais un recueil de cantiques plus digne de mention fut composé, pour l'usage des retraitants, par l'abbé Jean-Baptiste Boucher de Belleville, curé de Laprairie. Imprimé à Québec, en 1795, cet ouvrage contenait des chants extraits d'autres recueils, et aussi des mélodies nouvelles. C'est le premier livre de chants religieux préparé au Canada par un auteur canadien. Après l'avoir corrigé, on le réédita l'année suivante. A propos de cette deuxième édition, l'auteur écrit à l'imprimeur Neilson, en novembre 1797, ce qui suit : " 30 ou 40 fautes grossières qui se sont glissées dans votre impression, me font rougir de honte quand je rouvre le livre, et c'est là ce que vous appelez une édition corrigée: heureusement qu'on n'y trouve pas le nom de l'éditeur, il faudra bien que monsieur l'imprimeur porte tout le fardeau. " 
La troisième édition ne s'obtiendra qu'avec la promesse de corriger les fautes.

Le 15 septembre 1797, naît la bibliothèque circulante, qui fait imprimer un catalogue de 300 titres. Le propriétaire Thomas Carry spécifie que sa collection est chaste et cette publicité lui mérite d'être mentionné aujourd'hui dans les publications religieuses. Sans parler des Mandements des évêques, des sermons, des calendriers, des alma-

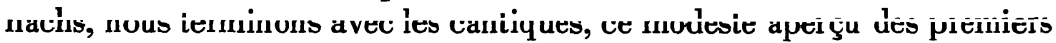
ouvrages religieux imprimés au Canada durant les premières années de l'imprimerie.

En fait, les éditions de ces ouvrages furent les plus importantes productions de l'imprimerie de cette époque. Elles fournirent un travail abondant aux imprimeurs anglais, protestants et athées et c'est grâce à eux si l'Église catholique canadienne a pu se procurer, pendant un siècle, les ouvrages nécessaires à son enseignement et à son apostolat. Comme on l'a vu, l'imprimerie se voua à l'enseignement populaire de la doctrine chrétienne. On y trouve, bien sûr, un résumé des vérités fondamentales que nous propose l'Église, mais on y voit aussi, à l'occasion, des aperçus pittoresques sur les mœurs et les pratiques du temps. On y voit que le clergé, comme le gouvernement, considérait l'instruction populaire plus dangereuse qu'utile.

En 1779, à la proposition du gouverneur Haldimand pour une bibliothèque publique qui devait loger au Palais de l'Évêque, une lettre de Mgr Montgolfier à Mgr Briand se lit ainsi : " Je vous assure, Monseigneur, que si je contribue à cet établissement, ce ne serait qu'à contre-cœur et par un motif de politique chrétienne. Je suis intimement persuadé que dans tous les établissements de l'imprimerie et de la bibliothèque publique, quoi qu'ils aient en eux quelque chose de bon, il y en a toujours plus de mauvais que de bon et qu'ils font plus de mal que de bien. "

Malgré l'immense service qu'ils rendent à la population, les imprimeurs sont regardés comme des libellistes et des fauteurs de désordre. Toute cette semence de littérature pieuse, dispersée à tout vent ne germait pas toujours en terre bien préparée pour la recevoir. Les voyageurs se plaignent de ne trouver dans les boutiques que des œuvres monacales.

Mais dans la Gazette du 10 janvier 1765, on trouve preuve plus convaincante des effets de cette germination.

Un mari, inventeur méconnu du courrier du cœur canadien, s'adresse à Monsieur l'imprimeur, en ces termes : "Je suis un pauvre infortuné Belitre, qui suis marié, à ma grande mortification, à une 
femme d'une piété et d'une vertu extraordinaires, ma Dame s'en va à l'église ou Chapelle voisine, elle confie le soin de la maison à un apprenti ignorant.

- Le pire, monsieur l'imprimeur, que la dévotion la rend d'une humeur diabolique. Dès qu'elle rentre dans la maison elle se retire dans sa chambre où elle passe son temps bien inutilement à lire quelques stupides recueils de prières fanatiques, ou à quelques sottes rapsodies dont on se sert dans nos couventicules, et là remplie de méditations et de malpropreté, elle croit s'acquitter du grand devoir de son état et ne pense pas une seule fois à son pauvre chien de mari. N'y a-t-il pas un moyen, monsieur l'imprimeur, pour guérir cette étrange maladie qui provient d'une trop grande dévotion.»

Fin de la citation et un dernier mot.

Cigale d'automne, j'ai dû emprunter tant de fois chez les historiens, mes voisins, que je veux leur dire publiquement ma reconnaissance. Évidemment, ce n'est ni de l'or ni de l'argent. Mais dans notre explosive évolution, la reconnaissance est peut-être la seule monnaie qui, parce qu'elle devient rare, se maintienne toujours à la hausse.

Madame Marcelle G. Gincras, Secrétaire de la Société Historique de Québec. 